\title{
COPD DETECTION USING THREE-DIMENSIONAL GAUSSIAN MARKOV RANDOM FIELDS BASED ON BINARY FEATURES
}

\author{
Yasseen Almakady ${ }^{\star} \quad$ Sasan Mahmoodi ${ }^{\star} \quad$ Michael Bennett ${ }^{\dagger}$ \\ ${ }^{\star}$ Department of Electronics and Computer Science, University of Southampton, United Kingdom \\ ${ }^{\dagger}$ Southampton NIHR Respiratory and Critical Care Biomedical Research Centre, \\ University Hospital Southampton NHS Foundation Trust, Southampton \\ Faculty of Medicine, University of Southampton, United Kingdom \\ Email: \{yham1n15,sm3y07,michael.bennett $\} @$ soton.ac.uk
}

\begin{abstract}
This paper proposes new descriptors based on three-dimensional Gaussian Markov random fields (3D-GMRF) for volumetric texture classification. The estimated parameters of 3DGMRF are decomposed into sign and magnitude components and then are encoded into a single binary code to describe the local texture. Our experiments on a synthetic dataset of volumetric texture show that this approach leads to significant reduction in descriptor size, while preserving the discriminative power of 3D-GMRF features. The descriptors proposed here demonstrate strong performance in distinguishing between healthy and chronic obstructive pulmonary disease (COPD) subjects, using a medical dataset. These descriptors are successfully employed to measure the differences between various groups from the medical dataset, in order to determine which group is at risk of COPD.
\end{abstract}

Index Terms - Volumetric texture classification, 3D texture, 3D-GMRF, COPD

\section{INTRODUCTION}

Texture is a fundamental property of many objects in our natural world, giving objects their unique appearance. This has led to the emergence of texture analysis as a vital topic in computer-vision and image-processing fields, playing a key role in many applications including medical image analysis [1]. Volumetric texture (or solid 3D texture) is the texture that can be found in 3D images and is indexed by $(x, y, z)$ $\in \mathbb{R}^{3}$. This type of texture has become increasingly available in the medical imaging domain, leveraging the rich information of organs' internal structure provided by different modalities such as magnetic resonance imaging (MRI) and highresolution computed tomography (HRCT). The common approach to handling volumetric texture is extracting textures from 2D slices of volumetric images. However, such an approach prevents sufficient exploitation of the valuable information provided by the third dimension in volumetric data.
The availability of volumetric images in medical imaging fields has led to the development of 3D methods to analyze this data for medical application [1]. In [2], a set of 3D filters is developed to extract texture features from 3D images for human ovarian tissue classification. The 3D texture-based method outperformed the $2 \mathrm{D}$ approach using the same tissue. This finding has also been concluded in [3], in which threedimensional Gaussian Markov random fields (3D-GMRF) were proposed to extract texture features from volumetric images of lungs and then were used for chronic obstructive pulmonary disease (COPD) detection. Such a 3D method demonstrates better results than using features extracted from 2D slices. The method presented in [4] extracts 3D features using 3D Riesz wavelets for usual interstitial pneumonia (UIP) classification. The extension of the well-known local binary pattern (LBP) [5] to 3D was introduced in [6] for achieving rotation invariance and was applied to discriminate between MRI of oxygenated and non-oxygenated brain tissues of newborn babies. The 3D grey-level cooccurrence matrix (3D-GLCM) was proposed in [7] to extract 3D texture features for volumetric texture classification.

Recently, deep-learning approaches like convolutional neural networks (CNN) have made remarkable contributions to image analysis, including image classification. Although $\mathrm{CNN}$ have become the choice for various computer-vison applications, including medical image analysis, CNN suffer from complications that limit their application in the medical imaging domain. In addition to their extensive memory and computation power, $\mathrm{CNN}$ require large annotated datasets for training and such datasets are difficult to obtain in the medical imaging domain, due the time and high costs required to annotate the datasets by experts $[8,9]$. This is further challenged by patient privacy concerns that restrict obtaining large medical datasets [10]. The limitation of using large datasets is partially overcome by employing transfer learning [11], although this is not always an optimal choice as it is influenced by different medical applications [8]. Therefore, approaches based on hand-crafted features could be a better 
choice for medical imaging analysis applications in which small amounts of training data are available.

In this paper, we propose an improved 3D-GMRF based on binary features for volumetric texture classification. We introduce new descriptors by encoding the 3D-GMRF estimated parameters $\boldsymbol{\alpha}_{v}$ into a binary code and employing them for COPD detection, which, to our knowledge, has not been reported in literature. Furthermore, the descriptors are successfully exploited to measure the change of texture in HRCT images of healthy non-smokers and healthy smokers groups to determine which group tends to be at risk of COPD. The remainder of the paper is organized as follows: Section 2 introduces our proposed method. The evaluation of the proposed method is presented in Section 3. Section 4 presents the medical application of the proposed method, while Section 5 concludes the paper.

\section{METHOD}

\subsection{Background}

GMRF has been applied for texture analysis by employing estimated parameters as features, which are then used for texture classification $[3,12,13]$ and segmentation $[14,15]$.

We define the 3D image as a set of grid points on 3D lattice $\Omega$ with size $H \times W \times D$ and indexed by $v=(i, j, k)$, where $\{1 \leq i \leq H, 1 \leq j \leq W, 1 \leq k \leq D\}$. The local conditional probability density function of the intensity value $g_{v}$ at location $v$ is defined by:

$p\left(g_{v} \mid y_{v+r}, r \in V_{v}\right)=\frac{1}{\sqrt{2 \pi \sigma^{2}}} \exp \left\{\frac{-1}{2 \sigma^{2}}\left(g_{v}-\lambda-\sum_{r \in V_{v}} \alpha_{r}\left(y_{v+r}-\lambda\right)\right)^{2}\right\}$

where $g_{v}$ is the central voxel at location $v$, surrounded by the neighbors $y_{v+r}$ located at relative position $r$. The neighborhood system is determined by $V_{v}$ and assumed here to be $P$ voxels sampled over a sphere surface centered at $v$ with radius $R$, so that $V_{v} \in\{\theta, \phi \mid 0 \leq \theta \leq \pi, 0 \leq \phi \leq 2 \pi\}$. The interaction parameters $\alpha_{r}$ measure the influence on $g_{v}$ by intensity values $y_{v+r}$, whereas $\lambda$ is the mean of the $3 \mathrm{D}$ patch since the 3D image is processed as a set of overlapping 3D patches (known as estimation cube $\Omega_{v} \subset \Omega$ ) to generate the 3D-GMRF model at each voxel. The 3D-GMRF model parameters $\alpha_{r}, \sigma^{2}$ in Equation (1) are unknown and need to be estimated by finding the best fit of model (1) to the texture data. The maximum likelihood estimation (MLE) is used here to estimate these parameters and it is computed by taking the partial derivative of a log-likelihood function, with respect to $\alpha_{r}$ and $\sigma^{2}$, and setting it to zero. The mean intensity $\lambda$ of $\Omega_{v}$ is computed separately and is subtracted from the intensity of $\Omega_{v}$ to avoid dealing with a non-linear equation system which is time consuming and may become unstable. Parameters $\alpha_{r}$ and $\sigma^{2}$ are then calculated from $\Omega_{v}$, whose mean is subtracted beforehand, leading to a set of linear equations for each voxel:

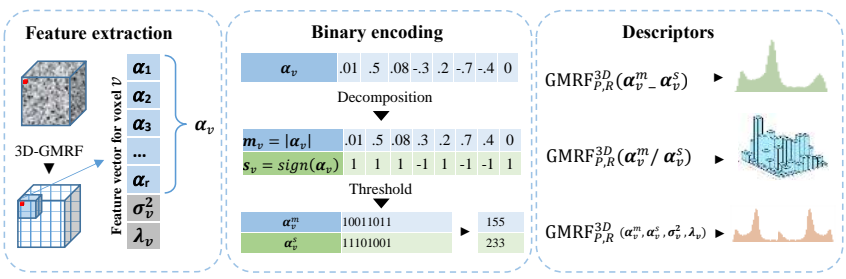

Fig. 1. Overview of our proposed method. (a) Features are extracted using 3D-GMRF. (b) $\boldsymbol{\alpha}_{v}$ is encoded into binary codes. (c) Descriptors are built in various ways.

$$
\begin{gathered}
\boldsymbol{\alpha}_{v}=\left(\sum_{v \in \Omega_{v}} \boldsymbol{y}_{v} \boldsymbol{y}_{v}^{T}+c^{2} I\right)^{-1}\left(\sum_{v \in \Omega_{v}} \boldsymbol{y}_{v} g_{v}\right) \\
\sigma_{v}^{2}=\frac{1}{\left|\Omega_{v}\right|} \sum_{v \in \Omega_{v}}\left(g_{v}-\boldsymbol{\alpha}_{v} \boldsymbol{y}_{v}\right)^{2}
\end{gathered}
$$

where $\boldsymbol{\alpha}_{v}=\operatorname{row}\left[\alpha_{r}\right]$ and $\boldsymbol{y}_{v}=\operatorname{col}\left[y_{v+r}\right]$ for $r \in V_{v}, I$ is the identity matrix with the same size as observation matrix $\boldsymbol{y}_{v} \boldsymbol{y}_{v}^{T}$, the superscript $T$ is the transpose operation, $c$ is a regularization parameter to ensure that the observation matrix is non-singular and invertible [3]. The parameters $\boldsymbol{\alpha}_{v}$ and $\sigma_{v}^{2}$ are estimated locally at each voxel $v$. The local estimation is performed by sliding an estimation cube $\Omega_{v}$ with size $w \times w \times w$ centered at each voxel $v$; then, the neighborhood sphere is slid inside $\Omega_{v}$ to collect overlapping samples that are used for parameter estimation. Once the parameter estimation is performed for the current voxel, $\Omega_{v}$ is moved to the next voxel and the previous procedure is carried out. This process produces a parameter volume in which each voxel is composed of a feature vector $\boldsymbol{f}_{v}=\left\{\boldsymbol{\alpha}_{v}, \sigma_{v}^{2}, \lambda_{v}\right\}$. These parameters are employed as features to describe the local texture. In our previous method proposed in [3], the descriptor is constructed by computing a histogram for each of calculated parameters $\boldsymbol{\alpha}_{v}, \sigma_{v}^{2}, \lambda_{v}$ over the entire image volume prior to being concatenated. However, this approach suffers from the expensive computational cost required by concatenating the histograms of multiple features, resulting in high-dimensional features. This is because increasing the number of neighbors leads to the increase of $\boldsymbol{\alpha}_{v}$, which eventually results in highdimensional descriptors. Inspired by [16], we propose an improvement to the 3D-GMRF model to reduce the number of features by encoding the parameter $\boldsymbol{\alpha}_{v}$ into a single code.

\subsection{Binary feature extraction for 3D-GMRF}

The feature vector $\boldsymbol{\alpha}_{v}$ is composed of the estimated parameters $\alpha_{r}$ that characterize local texture by measuring the strength of the relationship between the central voxel $g_{v}$ and its neighbor $\boldsymbol{y}_{v}$. The dimension of $\boldsymbol{\alpha}_{v}$ is equivalent to the number of neighbors distributed around $g_{v}$. Encoding $\boldsymbol{\alpha}_{v}$ into a single code, without losing its descriptive information, helps to significantly reduce the computation time 


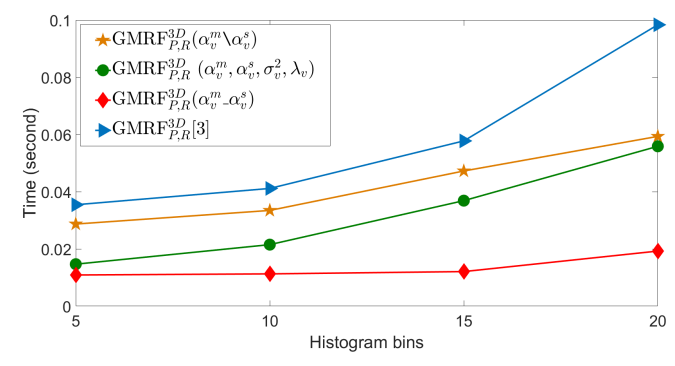

Fig. 2. Computation time (in seconds) for the proposed descriptors using different numbers of histogram bins.

required for texture classification. We used the binary encoding scheme, proposed in [5], to encode the sign and magnitude of $\boldsymbol{\alpha}_{v}$. The vector $\boldsymbol{\alpha}_{v}$ is first decomposed into two components represented by magnitude $\boldsymbol{m}_{v}=\left|\boldsymbol{\alpha}_{v}\right|$ and sign $\boldsymbol{s}_{v}=\operatorname{sign}\left(\boldsymbol{\alpha}_{v}\right)$ vectors defined at each voxel $v$. The next step is to encode $\boldsymbol{m}_{v}$ and $\boldsymbol{s}_{v}$ into single codes as follows:

$$
\begin{gathered}
\alpha_{v}^{m}=\sum_{p=0}^{P-1} t\left(\boldsymbol{m}_{v}(p), t_{m}\right) 2^{p} \quad t\left(x, t_{m}\right)=\left\{\begin{array}{l}
1, x \geq t_{m} \\
0, x<t_{m}
\end{array}\right. \\
\alpha_{v}^{s}=\sum_{p=0}^{P-1} t\left(\boldsymbol{s}_{v}(p)\right) 2^{p} \quad t(x)=\left\{\begin{array}{l}
1, x \geq 0 \\
0, x<0
\end{array}\right.
\end{gathered}
$$

where $P$ is the length of $\boldsymbol{\alpha}_{v}$, which is equivalent to the total number of neighbors surrounding $g_{v}, t_{m}$ is the mean of $\boldsymbol{m}_{v}$ over the entire volume. These encoded parameters $\alpha_{v}^{m}$ and $\alpha_{v}^{s}$ encompass discriminative information about the local texture and can be combined to construct descriptors by computing either the joint or concatenated histograms of $\alpha_{v}^{m}$ and $\alpha_{v}^{s}$, and are denoted as $\operatorname{GMRF}_{P, R}^{3 D}\left(\alpha_{v}^{m} \backslash \alpha_{v}^{s}\right)$ and $\operatorname{GMRF}_{P, R}^{3 D}\left(\alpha_{v}^{m}-\alpha_{v}^{s}\right)$, respectively. An additional descriptor can be constructed by exploiting the other estimated parameters of 3D-GMRF and is made from the concatenation of histograms of all extracted features, i.e., $\alpha_{v}^{m}, \alpha_{v}^{s}, \sigma_{v}^{2}, \lambda_{v}$, and is denoted as $\operatorname{GMRF}_{P, R}^{3 D}\left(\alpha_{v}^{m}, \alpha_{v}^{s}, \sigma_{v}^{2}, \lambda_{v}\right)$. Figure 1. shows the construction of the proposed descriptors.

\section{METHOD EVALUATION}

\subsection{Dataset, Settings, and Metrics}

We evaluated our method on the RFAI synthetic database for volumetric texture [18]. The database contains four datasets generated by four different methods, and each dataset is composed of four additional transformed subsets by applying rotation, noise, smoothing, and subsampling transformation to the normal subset of each dataset. Each of these subsets contains several classes and each class is composed of 10 volumetric images with a size of $64 \times 64 \times 64$. We used all datasets with their associated subsets except the rotate subset, since we were not interested in testing the rotation invariance property.

We applied our proposed method to each dataset in the RFAI database. The neighborhood scheme was chosen as a
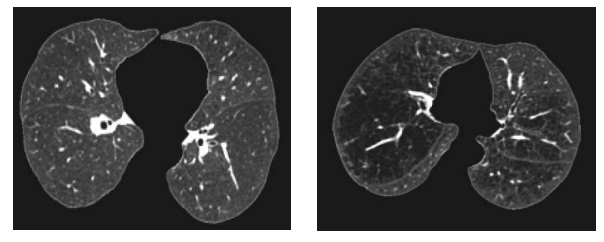

Fig. 3. Examples of a normal lung (Left) and a lung with COPD (Right).

sphere with $R=1$ and $P=42$ sampled voxels, where the size of the estimation cube $\Omega_{v}$ was selected as $w \times w \times w$ and $w=4 R+1$, leading to a size of $5 \times 5 \times 5$. The similarity between each of the two samples represented by the descriptors was estimated using $k$-nearest-neighbors $(k \mathrm{NN}, k=1$ ) with $L_{1}$ employed as a distance metric. For each dataset, we randomly selected $50 \%$ of each subset for training, while the remainder was kept for testing. This was repeated 100 times and then the mean and standard deviation were computed for all classification accuracies. The classification accuracy was calculated as the percentage of correctly classified samples using leave-one-out strategy to cover all samples. The performance of our proposed method was compared with the LBP method, based on the decision tree presented in [17] using the same RFAI dataset. Moreover, the method proposed here was compared with our previous work presented in [3].

The results presented in Table 1 demonstrate the discriminative power of our proposed descriptors. Although the three proposed descriptors perform well, $\operatorname{GMRF}_{P, R}^{3 D}\left(\alpha_{v}^{m}-\alpha_{v}^{s}\right)$, in particular, achieves good classification performance and outperforms the LBP-based method [17] involved in this experiment. One of the advantages of $\operatorname{GMRF}_{P, R}^{3 D}\left(\alpha_{v}^{m}-\alpha_{v}^{s}\right)$ is that it is not influenced by increasing the number of neighbors, as their equivalent parameters (i.e., $\boldsymbol{\alpha}_{v}$ ) are encoded into a single code, rather than computing the distribution of every single coefficient. This means that the dimension of the descriptor depends only on the number of histogram bins, which results in a stable computation time. This is particularly beneficial when multiresolution analysis is considered, as it requires more neighbors to be sampled around the central voxel. Figure 2 depicts the computation time required by each proposed descriptor to classify each descriptor of a dataset with 150 samples. This experiment was implemented using a MATLAB R2019b environment running on Intel Core i5 $3.3 \mathrm{GHz}$ processor with $8 \mathrm{~GB}$ of RAM. It can be observed that $\operatorname{GMRF}_{P, R}^{3 D}\left(\alpha_{v}^{m} \_\alpha_{v}^{s}\right)$ requires less time than other descriptors, combining high classification performance with less computation time compared with our method proposed in [3].

\section{APPLICATIONS FOR CHRONIC OBSTRUCTIVE PULMONARY DISEASE DETECTION}

In this experiment, the method proposed here is exploited to solve a real-world problem. COPD refers to a group of progressive lung diseases defined as a common, preventable, 
Table 1. Classification accuracies [\%] of our method and other methods using RFAI datasets.

\begin{tabular}{|c|c|c|c|c|c|c|c|c|c|c|c|c|c|c|c|c|c|}
\hline \multirow{3}{*}{ Descriptor } & \multicolumn{17}{|c|}{ Synthetic Texture Dataset } \\
\hline & \multicolumn{4}{|c|}{ Geometric } & \multicolumn{4}{|c|}{ Fourier } & \multicolumn{4}{|c|}{ Mixed texture } & \multicolumn{4}{|c|}{ Interpolated } & \multirow[t]{2}{*}{ mean \pm std } \\
\hline & noise & normal & smooth & subsampling & noise & normal & smooth & subsampling & noise & normal & smooth & subsampling & noise & normal & smooth & subsampling & \\
\hline $\operatorname{GMRF}_{42,1}^{3 D}\left(\alpha_{v}^{m} \_\alpha_{v}^{s}\right)$ & 99.77 & 100 & 99.82 & 98.92 & 99.96 & 99.04 & 98.68 & 97.81 & 99.78 & 99.92 & 99.34 & 100 & 93.80 & 95.95 & 93.78 & 96.90 & $98.34 \pm 2.13$ \\
\hline $\operatorname{GMRF}_{42,1}^{3 D}\left(\alpha_{v}^{m} \backslash \alpha_{v}^{s}\right)$ & 99.96 & 100 & 98.75 & 96.97 & 99.97 & 99.44 & 98.69 & 98.94 & 100 & 100 & 99.58 & 100 & 93.97 & 93.78 & 90.78 & 95.95 & $97.92 \pm 2.84$ \\
\hline $\operatorname{GMRF}_{42,1}^{3 D}\left(\alpha_{v}^{m}, \alpha_{v}^{s}, \sigma_{v}^{2}, \lambda_{v}\right)$ & 98.84 & 98.88 & 97.16 & 98.01 & 99.04 & 97.81 & 94.57 & 97.30 & 100 & 100 & 99.66 & 100 & 93.44 & 97.90 & 96.09 & 94.70 & $97.71 \pm 2.07$ \\
\hline $\mathrm{GMRF}_{42,1}^{3 D}[3]$ & 98.80 & 99.60 & 98.40 & 97.60 & 96.66 & 97.33 & 98.66 & 95.33 & 97.60 & 98.80 & 100 & 100 & 96.99 & 98.66 & 90.30 & 97.66 & $97.65 \pm 2.32$ \\
\hline LBP-based method [17] & 99.44 & 99.76 & 99.68 & 100 & 98.40 & 98.13 & 97.87 & 97.78 & 99.44 & 99.36 & 98.72 & 100 & 91.21 & 94.36 & 89.40 & 97.79 & $97.58 \pm 3.17$ \\
\hline
\end{tabular}

and treatable disease, characterized by persistent respiratory symptoms and airflow limitation due to airway and/or alveolar abnormalities [19]. The pathological changes of this disease affect lung texture appearance in HRCT scans. These textural changes can be used to characterize the disease and, hence, capturing these changes leads to the detection of COPD in HRCT scans.

Our medical dataset consists of 19 healthy individuals and 13 COPD patients, making a total of 32 subjects. Figure 3 shows examples of a normal lung and a lung with COPD. The HRCT image volumes were obtained from this dataset with size $256 \times 256 \times 256$ and then our proposed method was employed to distinguish between healthy individuals and COPD patients. The same settings and metrics described previously were used in this experiment. Classification performance was reported as classification accuracy, sensitivity/recall, and specificity. We compared our proposed method with two texture-based methods: 3D-GLCM [7], and the local parameters histogram (LPH) [13]. For 3D-GLCM, a set of texture features are derived from the 3D-GLCM by using different angular directions and distances for each HRCT scan. LPH is a 2D-GMRF-based rotation invariant method and it is included in this comparison to investigate the importance of texture found in 3D space for capturing more meaningful information. In this experiment, LPH was applied to each slice in the HRCT scans and the slices with the highest classification accuracy were selected to ensure a fair comparison.

The results of the classification performance of our proposed method and of other comparative methods are presented in Table 2. These results demonstrate the excellent discriminative power of our proposed method in distinguishing healthy individuals from COPD patients. The performance of our proposed method compared with LPH indicates the significance of texture features extracted from 3D space in providing more discriminative information that would help to distinguish between different samples. Another advantage of our method is that it does not require a large medical dataset for the training stage. The lack of large training datasets in the medical domain is a common case scenario. Therefore, our method could be appropriate for applications in field of medicine.

Further investigation was also carried out here to evaluate our method in identifying the differences between different groups of the COPD dataset, in order to understand which group tends to be at risk of COPD. The differences were iden-
Table 2. Comparison of our method with other texture-based methods using the COPD dataset

\begin{tabular}{|l|l|l|l|}
\hline \multicolumn{1}{|c|}{ Method } & \multicolumn{3}{c|}{ Results[\%] } \\
& Accuracy & \multicolumn{1}{c|}{ Sensitivity/Recall } & Specificity \\
\hline GMRFF $_{42,1}^{3 D}\left(\alpha_{v}^{m} \_\alpha_{v}^{s}\right)$ & $\mathbf{9 0 . 6}$ & $\mathbf{1 0 0}$ & $\mathbf{8 4 . 2 1}$ \\
LPH [13] & 81.25 & 100 & 68.42 \\
3D-GLCM [7] & 75.00 & 61.54 & 82.21 \\
\hline
\end{tabular}

Table 3. Differences between COPD, healthy smokers (HS) healthy non-smokers (HNS) groups of the COPD dataset.

\begin{tabular}{|l|lll|}
\hline Group & COPD-HNS & COPD-HS & HS-HNS \\
\hline Distance & 1.07 & 0.92 & 0.75 \\
\hline
\end{tabular}

tified by measuring the minimum Euclidean distance between the features of each two groups. Table 3 demonstrates that the healthy smokers group tends to be closer to COPD compared with the healthy non-smokers group. It is also noticeable that despite healthy smokers being closer to COPD than healthy non-smokers, they are still relatively close to each other, which is potentially because their lungs have not been significantly affected by COPD and, thus, the textural appearance is still identical to that in the lungs of healthy nonsmokers.

\section{CONCLUSION}

In this paper, we introduce new descriptors based on 3DGMRF for volumetric texture classification. The proposed descriptors are built by encoding the estimated parameters of 3D-GMRF into binary codes and computing their distribution over the whole volumetric image. We demonstrate that employing the encoding strategy for 3D-GMRF parameters improves classification performance using synthetic datasets of volumetric textures. Our new descriptors are successfully employed for COPD detection in the HRCT lung scans without requiring a large training dataset. Furthermore, we show that the descriptors can indicate healthy smokers' tendency to COPD. Future work aims to extend these descriptors to rotation invariant descriptors to be able to capture different texture patterns under random 3D rotations.

\section{Acknowledgments}

Imaging data were acquired from a previous study conducted by the Southampton NIHR Biomedical Research Centre, which was approved by the Southampton and South West Hampshire local research ethics committee (LREC number: 09/H0502/91) and the University Hospital Southampton Foundation Trust Research and Development Department. This research is funded by TVTC in Saudi Arabia. 


\section{REFERENCES}

[1] Adrien Depeursinge, Antonio Foncubierta-Rodriguez, Dimitri Van De Ville, and Henning Müller, "Threedimensional solid texture analysis in biomedical imaging: review and opportunities," Medical image analysis, vol. 18, no. 1, pp. 176-196, 2014.

[2] Bruce Wen, Kirby R Campbell, Karissa Tilbury, Oleg Nadiarnykh, Molly A Brewer, Manish Patankar, Vikas Singh, Kevin W Eliceiri, and Paul J Campagnola, "3d texture analysis for classification of second harmonic generation images of human ovarian cancer," Scientific reports, vol. 6, pp. 35734, 2016.

[3] Yasseen Almakady, Sasan Mahmoodi, Joy Conway, and Michael Bennett, "Volumetric texture analysis based on three-dimensional gaussian markov random fields for copd detection," in Annual Conference on Medical Image Understanding and Analysis. Springer, 2018, pp. 153-164.

[4] Adrien Depeursinge, Anne S Chin, Ann N Leung, Donato Terrone, Michael Bristow, Glenn Rosen, and Daniel L Rubin, "Automated classification of usual interstitial pneumonia using regional volumetric texture analysis in high-resolution ct," Investigative radiology, vol. 50, no. 4, pp. 261, 2015.

[5] Timo Ojala, Matti Pietikäinen, and Topi Mäenpää, "Multiresolution gray-scale and rotation invariant texture classification with local binary patterns," IEEE Transactions on Pattern Analysis \& Machine Intelligence, , no. 7, pp. 971-987, 2002.

[6] Leonardo Citraro, Sasan Mahmoodi, Angela Darekar, and Brigitte Vollmer, "Extended three-dimensional rotation invariant local binary patterns," Image and vision Computing, vol. 62, pp. 8-18, 2017.

[7] Elmoez Ben Othmen, Mounir Sayadi, and Farhat Fniaech, "3d gray level co-occurrence matrices for volumetric texture classification," in 3rd International Conference on Systems and Control. IEEE, 2013, pp. 833837.

[8] Nima Tajbakhsh, Jae Y Shin, Suryakanth R Gurudu, R Todd Hurst, Christopher B Kendall, Michael B Gotway, and Jianming Liang, "Convolutional neural networks for medical image analysis: Full training or fine tuning?," IEEE transactions on medical imaging, vol. 35, no. 5, pp. 1299-1312, 2016.

[9] Ashnil Kumar, Jinman Kim, David Lyndon, Michael Fulham, and Dagan Feng, "An ensemble of fine-tuned convolutional neural networks for medical image classification," IEEE journal of biomedical and health informatics, vol. 21, no. 1, pp. 31-40, 2016.
[10] Marysia Winkels and Taco S Cohen, "3d g-cnns for pulmonary nodule detection," arXiv preprint arXiv:1804.04656, 2018.

[11] Yutong Xie, Jianpeng Zhang, Yong Xia, Michael Fulham, and Yanning Zhang, "Fusing texture, shape and deep model-learned information at decision level for automated classification of lung nodules on chest ct," Information Fusion, vol. 42, pp. 102-110, 2018.

[12] Rama Chellappa and Shankar Chatterjee, "Classification of textures using gaussian markov random fields," IEEE Transactions on Acoustics, Speech, and Signal Processing, vol. 33, no. 4, pp. 959-963, 1985.

[13] Chathurika Dharmagunawardhana, Sasan Mahmoodi, Michael Bennett, and Mahesan Niranjan, "Rotation invariant texture descriptors based on gaussian markov random fields for classification," Pattern Recognition Letters, vol. 69, pp. 15-21, 2016.

[14] Yasseen Almakady, Sasan Mahmoodi Mahmoodi, and Michael Bennett, "Gaussian markov random fieldsbased features for volumetric texture segmentation," in 2019 IEEE Conference on Multimedia Information Processing and Retrieval (MIPR). IEEE, 2019, pp. 212215.

[15] Sasan Mahmoodi and Steve Gunn, "Snake based unsupervised texture segmentation using gaussian markov random field models," in 2011 18th IEEE International Conference on Image Processing. IEEE, 2011, pp. 3353-3356.

[16] Zhenhua Guo, Lei Zhang, and David Zhang, "A completed modeling of local binary pattern operator for texture classification," IEEE transactions on image processing, vol. 19, no. 6, pp. 1657-1663, 2010.

[17] Parmeet S Bhatia, Amit Kale, and Zhigang Peng, "Volumetric texture modeling using dominant and discriminative binary patterns," in Medical Imaging 2019: Image Processing. International Society for Optics and Photonics, 2019, vol. 10949, p. 109490H.

[18] Ludovic Paulhac, Pascal Makris, Jean-Yves Ramel, et al., "A solid texture database for segmentation and classification experiments.," in VISAPP (2), 2009, pp. 135-141.

[19] Claus F Vogelmeier, Gerard J Criner, Fernando J Martinez, Antonio Anzueto, Peter J Barnes, Jean Bourbeau, Bartolome R Celli, Rongchang Chen, Marc Decramer, Leonardo M Fabbri, et al., "Global strategy for the diagnosis, management, and prevention of chronic obstructive lung disease 2017 report. gold executive summary," American journal of respiratory and critical care medicine, vol. 195, no. 5, pp. 557-582, 2017. 